REVIEW ARTICLE OPEN

\title{
Ferroptosis: a cell death connecting oxidative stress, inflammation and cardiovascular diseases
}

\author{
Yi Yu ${ }^{1}$, Yuan Yan ${ }^{1}$, Fanglin Niu ${ }^{1}$, Yajun Wang ${ }^{1}$, Xueyi Chen ${ }^{1}$, Guodong Su${ }^{1}$, Yuru Liu ${ }^{1}$, Xiling Zhao ${ }^{1}$, Lu Qian ${ }^{2 凶}$, Ping Liu ${ }^{2 凶}$ and
} Yuyan Xiong ${ }^{1 \times}$

(c) The Author(s) 2021

Ferroptosis, a recently identified and iron-dependent cell death, differs from other cell death such as apoptosis, necroptosis, pyroptosis, and autophagy-dependent cell death. This form of cell death does not exhibit typical morphological and biochemical characteristics, including cell shrinkage, mitochondrial fragmentation, nuclear condensation. The dysfunction of lipid peroxide clearance, the presence of redox-active iron as well as oxidation of polyunsaturated fatty acid (PUFA)-containing phospholipids are three essential features of ferroptosis. Iron metabolism and lipid peroxidation signaling are increasingly recognized as central mediators of ferroptosis. Ferroptosis plays an important role in the regulation of oxidative stress and inflammatory responses. Accumulating evidence suggests that ferroptosis is implicated in a variety of cardiovascular diseases such as atherosclerosis, stroke, ischemia-reperfusion injury, and heart failure, indicating that targeting ferroptosis will present a novel therapeutic approach against cardiovascular diseases. Here, we provide an overview of the features, process, function, and mechanisms of ferroptosis, and its increasingly connected relevance to oxidative stress, inflammation, and cardiovascular diseases.

Cell Death Discovery (2021)7:193; https://doi.org/10.1038/s41420-021-00579-w

\section{Facts}

- Ferroptosis as a novel form of cell death differs from other cell death such as apoptosis, necroptosis, pyroptosis and autophagydependent cell death.

- Ferroptosis is closely related to iron and lipid metabolism and triggered by various physiological conditions and pathological stresses in human beings and animals.

- Ferroptosis connecting oxidative stress and inflammation plays key roles in the pathogenesis of cardiovascular diseases such as atherosclerosis, stroke, ischemia-reperfusion injury and heart failure.

\section{Open questions}

- What are the additional drivers for lipid peroxidation or ferroptosis, and the subcellular location of lethal lipid peroxides?

- What is the mechanism of lipid peroxidation leading to cell death?

- Upon ferroptosis, what are the intracellular components released to promote inflammatory responses and neighbor cell death?

- What are the adverse effects of targeting ferroptosis in cardiovascular diseases?

\section{INTRODUCTION}

Cardiovascular diseases (CVDs) are the leading causes of morbidity and mortality worldwide [1]. It is well-accepted that oxidative stress and inflammation play key roles in the pathogenesis of various cardiovascular disorders including atherosclerosis heart failure, ischemia/reperfusion injury and stroke, and the physiologic process of aging [2-4]. The imbalance between the production of reactive oxygen species (ROS) and the availability of antioxidants or free radical scavengers can cause oxidative stress, and that further activates an abundance of transcription factors and pro-inflammatory genes such as NFкB, p53, HIF-1 a, PPAR- $\gamma, \beta$-catenin/Wnt, and Nrf2 [5-7]. Activation of these transcription factors and genes recruit immune cells to secrete different cytokines and chemokines to trigger inflammation. Indeed, intracellular levels of ROS produced at a low level by normal aerobic metabolism are essential in normal cellular homeostasis and redox-dependent regulation of many signaling processes [8]. Whereas, a large number of ROS including superoxide $\left(\mathrm{O}_{2}{ }^{\cdot-}\right)$, hydroxyl radical $\left(\mathrm{OH}^{\circ}\right)$, perhydroxyl radical $\left(\mathrm{HO}_{2}{ }^{\circ}\right)$, and other types such as hydrogen peroxide $\left(\mathrm{H}_{2} \mathrm{O}_{2}\right)$, hypochlorous acid $(\mathrm{HOCl})$, and lipid peroxides $(\mathrm{ROOH})$ [9], is generated as by-products of cellular aerobic metabolism upon different physiological and pathological changes, once exceeding the capacity of the cellular reductase mechanisms, which can result in direct or indirect functional damage to different molecules such as proteins, nucleic acids, and lipids, and eventually lead to cell death $[10,11]$.

Cell death is of essence in the process of development, homeostasis, and pathogenesis of acute and chronic diseases, which tends to be accompanied with inflammation dysregulation, cell dysfunction, and tissue damage [12]. Programmed cell death

\footnotetext{
'Key Laboratory of Resource Biology and Biotechnology in Western China, Ministry of Education, School of Medicine, Northwest University, Xi'an 710069 Shaanxi, China. ${ }^{2}$ Department of Endocrinology, Xi'an No.3 Hospital, the Affiliated Hospital of Northwest University, Xi'an, Shaanxi 710018, P. R. China. \email: 2640933799@qq.com; 442711007@qq.com; yuyan.xiong@nwu.edu.cn

Edited by Ivano Amelio
}

Received: 4 May 2021 Revised: 6 June 2021 Accepted: 11 July 2021

Published online: 26 July 2021 
such as apoptosis, pyroptosis, and necroptosis play an important role in various physiological processes such as embryonic development, tissue homeostasis, and immunity in mammalian [13]. Ferroptosis is a new form of non-apoptotic cell death marked by the oxidative modification of phospholipid membranes via an iron-dependent mechanism [14]. Although the physiological function of ferroptosis is poorly understood, its implication in several human diseases such as tumorigenesis $[15,16]$, CVDs (e.g., atherosclerosis [17], ischemia-reperfusion injury (IRI) [18], cardiomyopathy [19, 20], and heart failure [21]) has been widely reported.

In recent years, accumulating studies demonstrate that ferroptosis is closely related to iron and lipid metabolism [22], which could be triggered by various physiological conditions and pathological stresses in human beings and animals [23]. As such, ferroptosis connecting oxidative stress and inflammation responses associated with iron and lipid metabolism plays a pathological role in CVDs. Here, we summarize the features, process, function, and mechanisms of ferroptosis, discuss its relevance with oxidative stress and cellular inflammation linking to cardiovascular disorders, raise the unsolved mysteries of ferroptosis, and provide new insights to consider the ferroptosis as the therapeutic target for the prevention and treatment of CVDs.

\section{FEATURES AND MECHANISMS OF FERROPTOSIS \\ Features of ferroptosis}

In 2012, ferroptosis was first termed by Stockwell Lab to define RAS-selective lethal small-molecule erastin-induced human fibrosarcoma cell death [23], which is iron-dependent, and morphologically and biochemically distinct from apoptosis, necrosis, autophagy-dependent cell death, and pyroptosis (Table 1). In ferroptotic cells, the intact cell membrane, cell swelling, reduction/ disappearance of mitochondria crista, and absent nuclear condensation or chromatin margination are observed [24]. Biochemically, depleted intracellular glutathione (GSH) and inactivated activity of glutathione peroxidase 4 (GPX4) lead to cell ferroptosis, since overproduced lipid peroxides cannot be abolished by the GPX4-catalyzed reduction reaction.

\section{The mechanisms of ferroptosis}

Currently, the exact mechanism of ferroptosis is still elusive, although there are several hypotheses. The mechanism may refer to the formation of lipid pore, similar to the proteinaceous pores observed in necroptosis and pyroptosis [25]. It appears that extensive oxidation of polyunsaturated fatty acid (PUFA)-containing phospholipids might modify membrane structure and increase membrane permeability, eventually resulting in plasma membrane rupture in response to accumulation of lipid-ROS, e.g., lipid hydroperoxides. Lipid hydroperoxides may promote the production of reactive toxic aldehydes such as 4-hydroxynonenals (4HNEs) and malondialdehydes (MDAs), which by crosslinking might inactivate essential cellular proteins to promote ferroptosis [16]. Several key regulators such as GPX4, iron, and ferroptosis suppressor protein 1 (FSP1) related signaling pathways were elucidated during the process of ferroptosis.

It is to note that apoptosis and necrosis inducers are incapable of triggering ferroptosis [26], suggesting that the mechanism underlying ferroptosis differs from that of apoptosis and necrosis. The ferroptosis inducers such as glutamate, erastin are able to drain GSH and inactivate the enzymatic activity of GPX4 via blocking the import of cystine by the cystine/glutamate antiporter (system $\mathrm{X}_{\mathrm{c}}{ }^{-}$) $[27,28]$ (Fig. 1). GSH, as an important ferroptosis suppressor and non-enzymatic antioxidant, provides a vital defense system to protect cells from different types of oxidative stress [29]. The ratio between GSH and its oxidative status GSSG usually indicates the level of cellular oxidative stress. As a member of the glutathione peroxidases (GPXs) family, GPX4 utilizes GSH as 


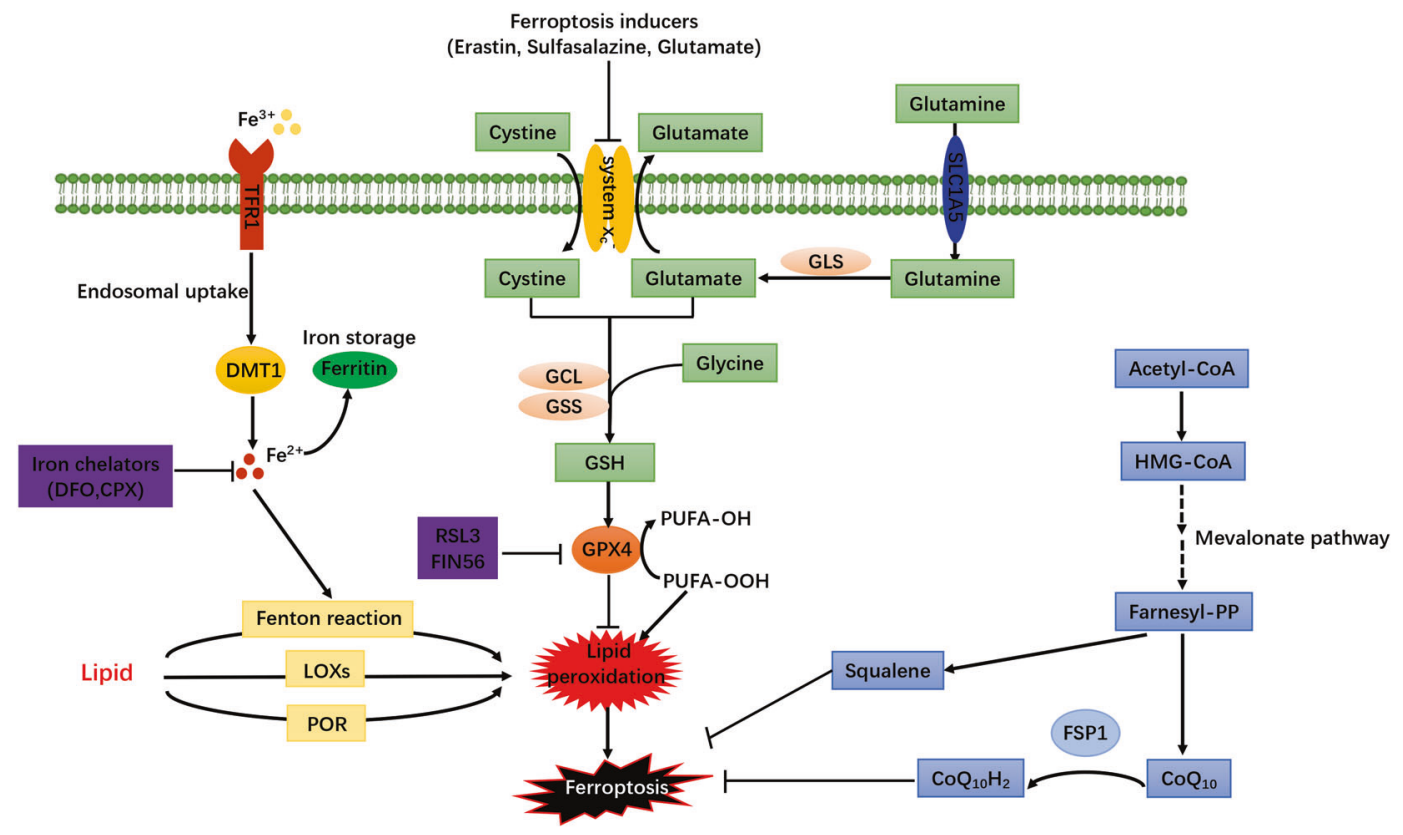

Fig. 1 The regulatory pathways of ferroptosis. Ferroptosis is triggered by the excessive lipid peroxidation (LPO). Cysteine and reduced glutathione $(\mathrm{GSH})$ availability are regulated by cystine-glutamate antiporter system $\mathrm{X}_{\mathrm{c}}{ }^{-}$or the transsulfuration pathway. The transsulfuration pathway is a metabolic pathway for the production of cysteine (Cys)-containing amino acids and the synthesis of GSH through the interconversion of cysteine and homocysteine. Glutamine can be transported into intracellular places by SLC1A5 transporter, and then converted into glutamate by glutaminase (GLS) to affect the GSH availability. GSH is a key cofactor of GPX4 for clearing lipid peroxides. The suppression of system $\mathrm{X}_{c}^{-}$by erastin or GPX4 activity by RSL3, which ultimately leads to cell ferroptosis. On the one hand, the peroxidation of PUFAs and excess irons is considered to be an important contributor. Iron uptake via the transferrin receptor-like TFR1 or degradation of ferritin iron stores enhances the labile iron pool, thereby cells are susceptible to ferroptosis via lipid hydroperoxides generation from the Fenton-like reaction. In addition, the mevalonate pathway is also involved in ferroptosis process by generating a series of biomolecules with potential anti-ferroptotic activity such as squalene, coenzyme Q10 (CoQ10), isopentenyl- pyrophosphate (IPP), and farnesyl pyrophosphate (FPP), which in part influences the ferroptosis occurrences. POR cytochrome P450 oxidoreductase, LOX lipoxygenase.

the electron donor to reduce toxic lipid hydroperoxides (lipid$\mathrm{OOH}$ ) in cell membranes to non-toxic lipid alcohols (lipid-OH) and water. Lipid-OOHs are unstable, and can be broken down to reactive compounds like malonaldehyde, hexanal, and 4-hydroxynonenal, etc., which are capable of serving as "oxidative stress second messengers" because of their longed half-life and ability to diffuse from their formation site [30, 31]. From this point of view, the direct GPX4 inhibitor such as RSL3 could ultimately promote overwhelming lipid-ROS generation and induce ferroptosis. The cystine-glutamate antiporter system $\mathrm{X}_{c}{ }^{-}$imports the extracellular cystine into the cytoplasm while it exports intracellular glutamate into the extracellular space. Cystine can be sequentially converted into GSH, which is indispensable in maintaining the intracellular redox balance as it can be utilized by GPX4 as a cofactor to alleviate lipid peroxidation (LPO) [32, 33] (Fig. 1). As a result, GSH deficiency, inactivation of GPX4 with ferroptosis inducer, or with the direct GPX4 inhibitor such as RSL3 ultimately promotes overwhelming lipid-ROS generation that leads to ferroptosis.

Ferroptosis is an iron-dependent cell death, and the normal inhibitors for apoptosis or necrosis can not prevent this process, but iron chelators work. Accumulated intracellular iron is another key to execute ferroptosis [14, 34] (Fig. 1). The hydroxyl radical $\left({ }^{\circ} \mathrm{OH}\right)$ is the most reactive radicals that can be produced through the Fenton reaction and Haber-Weiss reaction from hydrogen peroxide by catalysis of metal species (iron, copper) [35, 36]. Extracellular $\mathrm{Fe}^{3+}$, binding with transferrin, can be imported into cytoplasm through transferrin receptor (TFR). Then intracellular $\mathrm{Fe}^{3+}$ form is transferred into the endosome and reduced to $\mathrm{Fe}^{2+}$ by iron oxide reductase STEAP3. The reduced form $\mathrm{Fe}^{2+}$ is transported into a labile iron pool from the endosome by the divalent metal transporter 1 (DMT1) [37] (Fig. 1). Excess $\mathrm{Fe}^{2+}$ in cells will initiate non-enzymatic LPO through Fenton reaction.
Hydroxyl radical $(\cdot \mathrm{OH})$ directly generated from the Fenton reaction abstracts a hydrogen from PUFAs, forming a phospholipid carboncentered radical $(\mathrm{PL} \cdot)$, and proceeding with the oxygen addition to peroxyl radical (PL-OO.) to yield a phospholipid hydroperoxide $(\mathrm{PL}-\mathrm{OOH})$. Then accumulated lipid hydroperoxide is further converted to an alkoxyl radical (PL-O) in the presence of ferrous iron, which subsequently reacts with adjacent PUFAs to initiate another lipid radical chain reaction [16]. Furthermore, iron is also involved in the enzyme-mediated peroxidation process by lipoxygenases (LOX) [38], a family of lipid-peroxidizing enzymes, that catalyzes the peroxidation of PUFAs to generate lipid peroxyl radical [39]. It is to note that both the cellular lipid hydroperoxides mediated by LOXs enzyme and autoxidized peroxyl radicalmediated LPO may contribute to initiate ferroptosis [38, 40]. Therefore, accumulated intracellular iron is a fuse to trigger ferroptosis.

Except for canonical glutathione-based GSH-GPX4 axis, FSP1dependent pathway antagonizing ferroptosis has been recently uncovered. Bersuker et al. and Doll et al. coincidentally found that FSP1, previously known as apoptosis-inducing factor mitochondria-associated 2 (AIFM2), was an effective ferroptosisresistance factor, and protected cells from GPX4 deletion-induced ferroptosis [41, 42]. Myristoylated FSP1 was recruited to the plasma membrane, and catalyzed the reduction of ubiquinone $\left(\mathrm{CoQ}_{10}\right)$ by using $\mathrm{NAD}(\mathrm{P}) \mathrm{H}$ to form ubiquinol that functioned as a radical-trapping antioxidant to terminate LPO and finally suppressed ferroptosis. Thus, various biomolecules such as squalene and $\mathrm{CoQ}_{10}$ generated from mevalonate pathway also show potential roles in anti-ferroptosis [42-44] (Fig. 1). Nevertheless, a most recent study showed another potential mechanism of ferroptosis resistance for FSP1 independent of ubiquinol production that FSP1 modulates LPO through an ESCRT-III dependent 
membrane repair mechanism [45]. Moreover, beyond the role of tumor suppressor protein p53 in apoptosis, autophagy, and cell cycle, emerging evidence suggests that it sensitizes cells to ferroptosis via the regulation of cystine metabolism and ROS responses [15].

\section{FERROPTOSIS CROSS-LINKING ROS AND INFLAMMATION}

Various types of regulated cell death (RCD) are implicated in the regulation of inflammation. In recent years, a concept of "necroinflammation" was raised and defined to describe the inflammatory response to necrotic cell death in a living organism $[46,47]$. As a consequence of cell death, its intracellular components are released as pro-inflammatory damage-associated molecular patterns (DAMPs) such as ATP, nucleotides, histones, and high-mobility group protein B1 (HMGB1) and proinflammatory cytokines, such as interleukin-1a (IL-1a), IL-33, and interferon- $\gamma$ (IFN $\gamma$ ) upon plasma membrane rupture, which triggers the innate immune system [48]. Following an initial event of RCD, DAMPs accumulation can trigger tissue inflammation which can further promote RCD to form a self-amplified circuit of necro-inflammatory loop that leads to exaggerated cell death and inflammation, eventually resulting in tissue damage and organ dysfunction [48, 49]. Unlike the in-depth understood roles of immunologically non-silent types of regulated necrotic cell death such as necroptosis and pyroptosis, the study of necroinflammation in ferroptosis is still in the early stages [50]. Ferroptotic cell death is also capable of releasing DAMPs that promote sterile inflammation and the development of numerous inflammatory diseases, for example, via its receptor for advanced glycation endproducts (RAGE) to activate NF-KB [51, 52] (Fig. 2).

In addition, inflammation is closely associated with oxidative stress, which are able to trigger a series of transcription factors such as Nrf2, NF-kB1, and pro-inflammatory cytokines such as TNF$a$, resulting in the differential expression of inflammatory cytokines, chemokines, and anti-inflammatory molecules [7, 53]. Redundant ROS induces oxidative stress and consume intracellular antioxidants, which further exacerbates the production of LPO and inflammatory responses in a vicious circle. Moreover, LPO drives the increase in modified LDL, which in part promotes inflammation via macrophage polarization (Fig. 2). Therefore, LPO can be observed in multiple physiological conditions such as cell death and inflammatory response in the pathophysiology of various diseases.

GSH and GPX4, the key regulators of ferroptosis, are also crucial in mediating inflammatory responses. GSH, as the most abundant antioxidant, is necessary to buffer rising ROS and prevent inflammation-associated cellular damage [54]. Disruption of GPX4 in a human carcinoma cell line can up-regulate the expression of 12-lipoxygenase and cyclooxygenase 1 (COX1) that triggers inflammatory responses $[55,56]$. On the contrary, GPX4 activation suppressed ferroptosis and inflammatory responses via blunting arachidonic acid (AA) oxidation and NF-KB pathway activation, accompanying by decreased ROS generation [57]. AA, an unsaturated fatty acid, released from the cell membrane in response to various cytokines, peptides and growth factors [58], can be metabolized by COX, LOX, and cytochrome P450 (CYP450) monooxygenases to synthesize bioactive inflammatory mediators such as prostaglandins (PGs), leukotrienes (LTs), epoxyeicosatrienenoic acids (EETs) and hydroxyeicosatetraenoic acids (HETEs) [59] (Fig. 2), which play important roles in inflammatory responses and many physiological processes [60-62]. LOXs have been demonstrated as central players in ferroptosis-associated accumulation of the products of LOX catalysis [38].

Iron, a vital regulator of ferroptosis, also participates in the regulation of immune system. Iron metabolism in inflammation has been well characterized and intracellular iron overload promotes M1 macrophage polarization [63]. Handa et al. reported

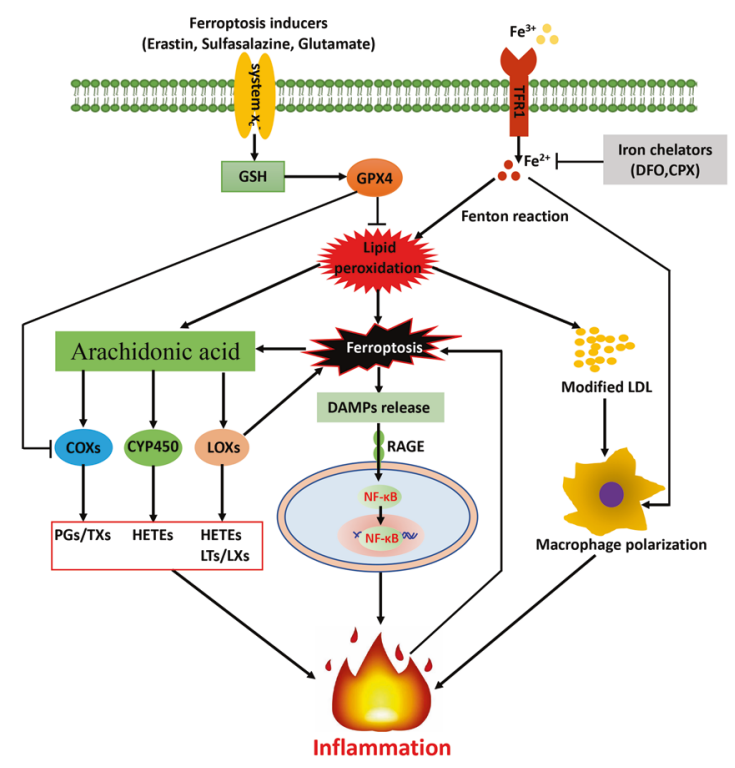

Fig. 2 Cross-talk between ferroptosis and inflammation. ROSinduced oxidative stress exacerbates the production of lipid peroxidation (LPO). LPO-induced ferroptotic cell death results in the release of damage-associated molecular patterns (DAMPs) from dying cells, which may promote sterile inflammation through the receptor for advanced glycation end products (RAGE) to activate NF$\kappa B$. On the other hand, lipid peroxidation induced the increase in modified LDL partially contributes to inflammation activation via macrophage polarization. GPX4, the core regulator of ferroptosis, its activation suppressed ferroptosis and inflammatory responses via inhibiting arachidonic acid (AA) oxidation and NF- $\mathrm{KB}$ pathway activation. Moreover, GPX4 can up-regulate the expression of 12lipoxygenase and cyclooxygenase 1 (COX1) that trigger inflammatory responses. AA, an unsaturated fatty acid and released from the cell membrane in response to various cytokines, peptides, and growth factors, that can be metabolized by cyclooxygenases (COXs), lipoxygenase (LOX), and cytochrome P450 (CYP450) monooxygenases to synthesize bioactive proinflammatory mediators such as prostaglandins (PGs), leukotrienes (LTs), lipoxins (LXs), hydroxyeicosatetraenoic acids (HETE) and hydroperoxyeicosatetraenoic acids (HPETE) that promote inflammatory responses. Finally, another vital regulator of ferroptosis, iron also participates the regulation of immune system via modulating M1 macrophage polarization.

that dietary iron overload in mice was able to induce hepatic macrophage $\mathrm{M} 1$ activation, and was also accompanied by hepatic fibrogenesis and steatohepatitis [64]. DIBI, a novel iron-chelator, was found to reduce the levels of inflammatory mediators and restore functional capillary density in the intestinal muscle layer for sepsis treatment $[65,66]$. Interestingly, iron was found to alter the balance between $\mathrm{M} 1 / \mathrm{M} 2$ macrophage polarization, leading to macrophage-driven inflammation and fibrogenesis in nonalcoholic fatty liver disease (NAFLD) [64].

\section{FERROPTOSIS IN CVDS}

\section{Ferroptosis in atherosclerosis}

In the past decade, several cell death types such as apoptosis, pyroptosis, necroptosis, and ferroptosis, are reported to contribute to atherosclerotic lesions formation during all stages. Liu et al. and our group reported that macrophage and VMSCs apoptosis is necessary to reduce macrophage burden and stabilize the atherosclerotic plaque within the developing lesion in Apo $\mathrm{E}^{-1-}$ mice $[67,68]$. In addition to apoptosis, pyroptosis and necroptosis are also implicated in advanced atherosclerotic lesions and ruptured human plaques [69]. In Apo $\mathrm{E}^{-/-}$mice, inhibition of pyroptosis and necroptosis is able to ameliorate atherogenesis and reduce necrotic core size [70-72]. Ferroptosis, a newly 
discovered form of iron and LPO-dependent cell death, has been also implicated in atherogenesis. Bai et al. showed that inhibition of ferroptosis by ferrostatin-1 (Fer-1) administration alleviates high-fat diet (HFD)-induced atherosclerosis in Apo $E^{-/-}$mice through reducing LPO in mouse aortic endothelial cells [17].

The PUFA-OOH, the main source of LPO, may trigger endothelial ROS increase, nitric oxide (NO) decrease, chronic inflammation in macrophages, and formation of foam cells, and eventually contributes to the formation of atherosclerotic lesions. Besides, ferroptosis-associated peroxidation of LDL in the endothelium is capable of causing endothelial dysfunction and macrophages activation. Prior to discovery of ferroptosis, overexpression of GPX4 was reported to scavenge extra ROS and phospholipid hydroperoxides, and significantly slow down the progression of atherosclerotic plaque in Apo $\mathrm{E}^{-/-}$mice [73]. Apart from the GPX4, extensive studies have shown that iron was also involved in the development of atherosclerotic lesion through affecting LPO in vitro and in vivo [74, 75]. Iron-catalyzed free radical reactions cause oxidation of LDL in endothelial cells, smooth muscle cells, or macrophages, which may be risk factors in the formation of atherosclerotic lesions [76]. Oxidized-low density lipoprotein (Ox$\mathrm{LDL}$ ) induced ferroptosis and iron accumulation were observed in mouse aorta endothelial cells, and this event can be reversed by ferroptosis inhibitors [17]. Transferrin, the major plasma ironbinding molecule, interacts with transferrin receptor protein 1 (TFR1) to deliver extracellular $\mathrm{Fe}^{3+}$ into cells, leading to iron pool overload and increased cell susceptibility to ferroptosis [77]. Ferritin and LDL-cholesterol levels showed a synergistic association with the incidence of CVD and death [78]. As LPO as well as iron deposition are prominent features of atherosclerotic plaques [79], it is rational to deduce that ferroptosis in ECs, VMSCs, and macrophages may be implicated in the progression of atherosclerotic plaque destabilization. Taken together, ferroptosis connecting LPO, inflammation, and iron storage may play a crucial role in the progression of atherosclerosis (Fig. 3).

\section{Ferroptosis in ischemia/reperfusion injury}

IRI refers to the tissue damage caused by blood supply restores to ischemic tissue (reperfusion) after a period of ischemia. Reperfusion of organ is indispensable event to rescue tissue from the long-term oxygen deprivation. However, restoration of circulation is accompanied with inevitable cell death, oxidative stress damage, inflammation and programmed cell death, and eventually results in tissue injury such as myocardial infarction (MI) [80]. As such, IRI is a distressing issue at the beginning of organ transplantation era and impedes the recovery of organ transplanted patient. During the process of ischemic tissue restoring to aerobic metabolism, free radicals might be generated via oxidizing various cellular components [81]. Garci-Gil et al. have shown that LPO is the major contributor to cause ischemia-reperfusion oxidative injury on a pig pancreas transplantation model [82]. Another study reported that reperfusion of ischemic cardiac tissue enhanced the concentration of LPO product 4-HNE, contributing to the declines in mitochondrial function [83]. In 2013, Linkermann et al. found combined inhibition of mitochondrial permeability transition (MPT)-induced regulated necrosis (RN) and receptorinteracting protein kinase (RIPK)1-mediated necroptosis offered protective effects in IRI model [84]. Further study based on an artificial model of GPX4 deletion in the proximal tubules indicated that knockout of GPX4 caused cell death in a pathologically relevant form of ferroptosis and spontaneous tubular necrosis, and the inhibition of ferroptosis by liproxstatin-1 was able to mitigate ischemia/reperfusion-induced tissue damage [85]. Moreover, a variety of studies have shown that ferroptosis is involved in IRI in the liver [86], brain [87], and heart [88]. More recently, several studies have uncovered some interesting finding that inhibition of ferroptosis ameliorated IRI in different tissues. Inhibition of ferroptosis by ferrostatin-1 (Fer-1) was shown to reduce infarct

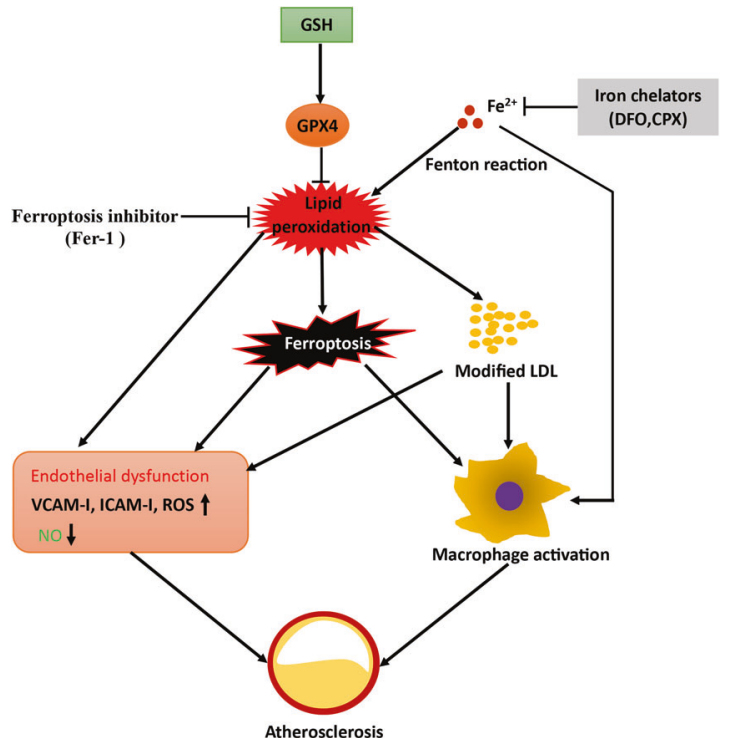

Fig. 3 The role of ferroptosis in atherosclerosis. Atherosclerosis is a chronic inflammatory disorder. Ferroptosis in endothelial cells and macrophages can lead to endothelial dysfunction (e.g., VCAM-I, ICAM-I, ROS increase, NO decreases) and macrophage activation, which contributes to the generation of atherosclerosis. Ferroptosisassociated peroxidation of LDL in the endothelium is capable of causing endothelial dysfunction and macrophages activation. The PUFA-OOH, the main source of lipid peroxidation (LPO) may also trigger endothelial ROS increase, nitric oxide (NO) decrease, chronic inflammation in macrophages and formation of foam cells. Ironcatalyzed free radical reactions cause not only ferroptosis but also the oxidation of LDL in endothelial cells, smooth muscle cells or macrophages, which are also involved in the formation of atherosclerotic lesions.

size, improve left ventricular (LV) systolic function, and attenuate LV remodeling in myocardial IRI via lowering the levels of proferroptotic LPO and reducing cardiomyocyte cell death, providing evidence that ferroptosis regulates cardiomyocyte cell death in the context of $\mathrm{MI}$ [89]. In addition, a single cell undergoing NADPH-depleting ferroptosis may deplete the NADPH concentration of neighbor cells that are connected to the cytoplasm of this cell, which causes the synchronized necrosis of neighbor cells in a functional organ [90]. As this phenomenon of synchronized RN is predominantly mediated by ferroptosis, IRI may critically involve the spreading of necrotic lesions within functional units such as a tubule or maybe between cardiomyocytes [91].

Iron overload is another factor to promote tissue oxidation during ischemia and reperfusion, and becomes a novel risk factor for predicting hepatic I/R injury in liver transplantation and lung oxidative injury with ischemia/reperfusion in rat lungs [18, 92-94]. As such, iron-dependent ferroptosis is doomed to affect the process of IRI (Fig. 4). Yamada et al. found that I/R was able to lead to liver damage, LPO as well as upregulation of the ferroptosis marker Ptgs2 in a murine model, and all of these effects were significantly alleviated by ferroptosis-specific inhibitor ferrostatin-1 and iron chelator deferoxamine [18]. In addition, tissue ischemia and reperfusion are accompanied with pro-inflammation, thus ferroptosis-associated inflammatory responses may play an unexpected role in IRI. Collectively, ferroptosis contributes to the development of tissue I/R induced injury (Fig. 4), and presents a potential therapeutic target for the prevention of organ $I / R$ damage, e.g., solid organ transplantation.

\section{Ferroptosis in stroke}

Stroke is one major cause of death worldwide, and its incidence including ischemia or hemorrhage leads to the interruption or 


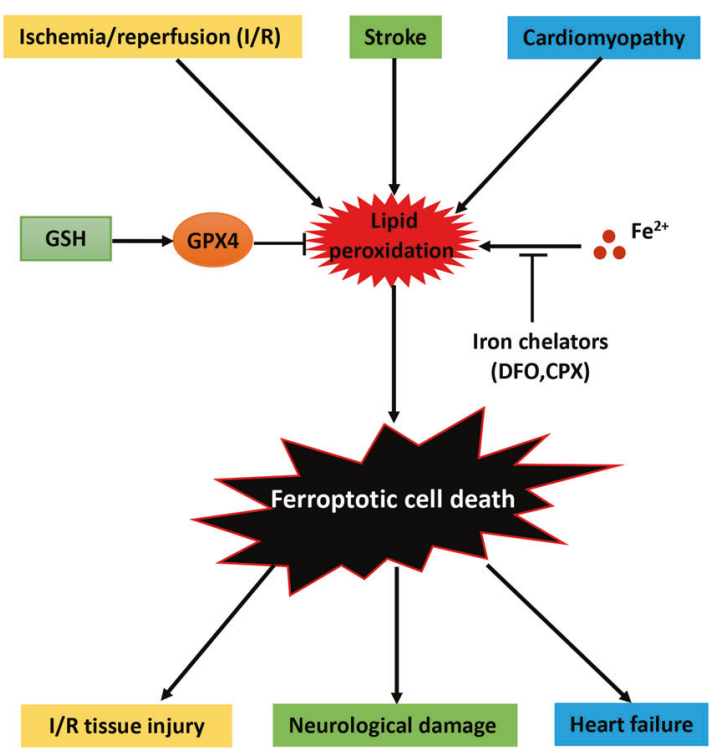

Fig. 4 The role of ferroptosis in I/R injury, stroke and heart failure. Ischemia-reperfusion (IR), stroke, and cardiomyopathy are accompanied with not only inevitable mitochondrial dysfunction, lipid peroxidation (LPO) and inflammation but also ferroptotic cell death, which results in tissue injuries such as ischemia-reperfusion injury, neurological damage, and heart failure that can be ameliorated by inhibition of ferroptosis via increasing GSH levels, GPX activity or iron chelation.

reduction of body blood supply to brain. Ferroptosis has been shown to be implicated in the pathological cell death associated with stroke [27]. Alim et al. reported that selenium suppresses ferroptotic cell death to protect neurons from hemorrhagic or ischemic stroke damage by activating the transcription factors TFAP2c and Sp1 [95]. Iron has an fundamental role in many physiological processes, including erythropoiesis, immunity, and oxidative metabolism [96]. Iron is also a key component of cytochromes $\mathrm{a}-\mathrm{c}$ of the oxidative chain, and responsible for the production of adenosine triphosphate (ATP) [97]. However, disturbances in iron homeostasis have been linked to neuronal damage following ischemic injury [98]. By catalyzing Fenton reactions that convert superoxide and hydrogen peroxide into reactive toxic hydroxyl radicals, iron can induce ferroptosis and subsequent neuronal injury [99]. As such, iron is causally linked to LPO production such as lipid hydroperoxides that promotes ferroptosis, and to conditions that predispose to ischemic stroke [100] (Fig. 4). Both basic and clinical evidence validate that LPO dysregulation mediates stroke injury. LPO products such as MDA, thiobarbituric acid-reactive substances and 4-hydroxynonenal already showed the obvious augmentation and detrimental effects in ischemic stroke [101-103]. Clinical study also exhibited that the mean values of lipid hydroperoxidesin in the plasma of stroke patients were $\sim 2.5$-fold higher than controls [104]. Moreover, the rupture of the blood vessel wall in hemorrhagic stroke results in the accumulation of hemoglobin and heme iron at the extracellular milieu and subsequent iron-induced LPO and ferroptosis, thereby furtherly exacerbating the stroke-induced brain injury.

\section{Ferroptosis in heart failure}

Heart failure occurs when the ability of heart pumping blood to the rest of our body is seriously compromised. Cardiomyopathy is a major factor to lead to heart failure. The loss of terminally differentiated cardiomyocytes is a lethal pathogenic factor in the pathophysiological process of cardiomyopathy. Whereas, the molecular mechanisms of cardiomyocyte death are still not well elucidated.

It was demonstrated that ferroptosis is highly correlated with cardiomyocyte death, and mammalian target of rapamycin (mTOR) plays an important role in protecting cardiomyocytes against excess iron and ferroptosis by inhibiting the production of lipid-derived ROS [105]. In the most recent study, Wang et al. group presented that iron overload-induced ferroptosis was a major contributor to doxorubicin (DOX) induced cardiomyopathy, and treatment with the ferroptosis inhibitor ferrostatin-1 remarkably improved the survival of DOX-treated mice. Moreover, they figured out that heme oxygenase-1 (HMOX1) was the major culprit for iron release in DOX-induced cardiotoxicity and provided intriguing insights for the first time into the role of ferroptosis in cardiac cell death linked to cardiomyopathy $[19,106]$. Next, Wang et al. further revealed that ferrostatin-1adminstration rescued high-iron diet-induced severe cardiac injury and hypertrophic cardiomyopathy, with molecular features typical of ferroptosis, including reduced GSH levels and increased LPO. Finally, they found that the expression of ferroptosis regulator SLC7A11 transporting cystine (the precursor of GSH) into the cytosol was suppressed in conditional Fth knockout cardiomyocytes, and overexpressing SLC7A11 in cardiomyocytes selectively increased the levels of GSH that prevented cardiac ferroptosis [20]. Puerarin, one of the most abundant phytoestrogens with antioxidant activity, could improve myocyte loss during heart failure induced by pressure overload through ferroptosis suppression in rat model [21]. In addition, the classical ferroptosis inducer erastin was also reported to damage cardiomyocytes by initiating ferroptosis, while ENPP2, a lipid kinase generating the lipid mediator lysophosphatidic acid, could protect cardiomyocytes from the erastin-induced ferroptosis by decreasing ROS generation [107]. Moreover, inhibition of endogenous expression of TLR4 and NADPH oxidase 4 that were upregulated in rat cardiac tissue after heart failure, showed heart failure relief by suppressing ferroptosis in cardiac cells [108]. These studies disclose ferroptotic cell death is implicated in the regulation of heart failure (Fig. 4). However, the molecular mechanisms of ferroptosis in cardiomyocytes still require further investigation.

\section{THERAPEUTIC STRATEGY TARGETING FERROPTOSIS IN CVDS}

Ferroptosis connecting oxidative stress, inflammation, and cell death, plays a pathophysiological role in CVDs. Therefore, trapping ferroptosis is promising strategy to treat ferroptosis-associated cardio- and vascular- disorders. To study the potential role of ferroptosis in vivo, effective and specific small molecular ferroptosis inhibitors have been identified and can be categorized into iron chelators, lipophilic radical-trapping antioxidants, and system $X_{c}^{-}$[109].

\section{Iron chelators}

As iron participates in the generation of lipid-ROS and ferroptosis occurrence [110], the medical use of chelators in humans in the prevention of ferroptosis-associated CVDs (e.g., atherosclerosis, IRI and cardiomyopathy) is optimistic [111]. Deferoxamine (DFO) is a clinically approved iron chelator with a very high affinity to bind with the trivalent ferric ion to remove iron [111]. Various studies showed that DFO reduced cytosolic ROS production and ferroptotic cell death [26]. In vivo, DFO also shows cardioprotective effects by inducing ischemic preconditioning-like effects in $\mathrm{I} / \mathrm{R}$ models, and reduction of mice atherosclerotic lesion by inhibiting inflammation and impairing nitric oxide action [112-114]. In type 2 diabetic rat model, inhibition of ferroptosis by DFO treatment may prevent post-stroke cognitive impairment [115]. DFO administration showed cardioprotective effects in $\mathrm{I} / \mathrm{R}$ models via inhibiting cytosolic ROS production and ferroptotic cell death [112]. Dexrazoxane (DXZ) is the only iron chelator drug approved 
by the FDA and shows cardioprotective effects with ferroptosis inhibition in animal models of doxorubicin-induced cardiomyopathy [19]. The clinical use of iron chelators in treating iron load disorders makes it a potential therapeutical strategy in the prevention of ferroptosis-related CVDs.

\section{GPX4 and GSH}

GPX4 is critical in preventing excess free radical generation and ferroptotic cell death [116], as it utilizes GSH as the electron donor to reduce toxic lipid hydroperoxides in cell membranes to the corresponding alcohols and water [117]. Ferroptosis is associated with GPX4 inactivation that leads to lipid oxidative stress. Conditional GPX4 knockout in myeloid lineage cells results in LPO-dependent caspase-11 activation and increase of $\mathrm{N}$-terminal gasdermin $\mathrm{D}$ (GSDMD) fragments, which triggers macrophage pyroptotic cell death and release of DAMPs such as high-mobility group box 1, alarmin and pro-IL-1 $\beta$ [118]. Taken together, it is worth to notice that the phenomenon of synchronized regulated pyroptosis might occur and be mediated by GPX4-dependent ferroptosis. Alternatively, GPX4 depletion initially triggers pyroptotsis and subsequently regulates inflammation and ferroptosis. In vivo, GPX4 deficiency in mice caused embryonic lethality, which may be correlated with increased ferroptotic cell death and enhanced LPO [119, 120].

In addition, an adequate supply of GSH is beneficial to attenuate ferroptosis. Thiol supplementation with GSH selectively improves human endothelial dysfunction by enhancing NO activity [121]. While mitochondria-specific transgenic overexpression of GPX4 in mitochondria is also able to decrease LPO and attenuate rat I/R injury [122]. Given GPX4 activity and GSH level that could modulate oxidative stress, thus any dysregulation of GSH and GPX4 might promote ferroptosis and lead to adverse cardiac disorders. Several nature products with antioxidant activity such as vitamins and vitamin precursors have shown efficient inhibitory effects of ferroptotic cell death and potential cardioprotective effects [116, 123]. Vitamin E supplements protect against atherosclerosis in vivo, this action might be through preventing ferroptosis by lowering the oxidation of $\operatorname{LDL}[124,125]$.

\section{Ferrostatins and liproxstatins}

Ferrostatins and liproxstatins are screened compounds that act as lipophilic antioxidants to inhibit LPO associated with GPX4 deficiency or inhibition induced ferroptosis. Ferrostatin-1 (Fer-1), one of the ferrostatin family, is the extensively researched compound that suppresses different inducers induced ferroptosis in a variety of cell lines, tissue types, and disease models [22]. At the most recent study, Bai et al. showed Fer-1 ameliorated HFDinduced AS lesion in Apo $\mathrm{E}^{-/-}$mice with the reduction of iron accumulation and LPO, and increase of expressions SLC7A11 and GPX4. Oxidized-low density lipoprotein (ox-LDL)-induced mouse aortic endothelial cells dysfunction can be significantly improved by Fer-1 treatment [17]. Moreover, Fer-1 also protects the heart against $\mathrm{I} / \mathrm{R}$ injury and doxorubicin-induced cardiomyopathy by inhibiting ferroptosis [19]. Not only that, the administration of Fer1 in vivo reduced neuronal death and improved neurologic function after intracerebral hemorrhage $(\mathrm{ICH})$ by inhibiting lipid ROS generation and COX-2 expression [87]. Liproxstatin-1 (Lip-1), the first identified molecule of liproxstatin class, suppresses ferroptosis by scavenging free radical to prevent PUFA oxidation $[85,126]$. Lip-1 treatment showed remarkable reduction in myocardial infarct sizes and maintained mitochondrial structural integrity and function against I/R injury by reducing VDAC1 levels and restoring GPX4 levels [127]. Therefore, screening a variety of small-molecule as ferroptosis inhibitors like Fer-1 and Lip-1 could be optimistic strategy for CVD drugs development in the future.

\footnotetext{
System $\mathbf{X}_{\mathbf{c}}^{-}$

System $\mathrm{X}_{\mathrm{c}}{ }^{-}$, a crucial negative regulator of ferroptosis, structurally consists of the twelve-pass transmembrane transport protein
}

cystine/glutamate transporter (SLC7A11) and the single-pass transmembrane regulator protein 4F2 cell-surface antigen heavy chain (SLC3A2), and is responsible for maintaining redox homeostasis by importing cystine to synthesize GSH [128, 129]. Inhibiting system $\mathrm{X}_{\mathrm{c}}^{-}$with compounds such as sorafenib and sulfasalazine can promote ferroptosis, while overexpression of system $\mathrm{X}_{c}^{-}$components by contrast is capable of diminishing erastin-induced ferroptosis [23]. Fang et al. showed conditional Fth-deficient cardiomyocytes suppressed the expression of the ferroptosis regulator SLC7A11, and overexpressing SLC7A11 selectively in cardiomyocytes increased GSH levels and suppressed cardiac ferroptosis, indicating that targeting SLC7A11 might offer new therapeutic opportunities for treating and/or preventing cardiac complications [20].

\section{POTENTIAL RISK OF FERROPTOSIS-INDUCING AGENTS TO CARDIOVASCULAR SYSTEM}

Over the past a few years, emerging evidence indicates that induction of ferroptotic cell death by ferroptosis-inducing agents in cancer cells may provide a potent strategy for cancer therapy. Some ferroptosis-inducing (FIN) compounds from improved analogs of erastin with increased solubility and selectivity showed potency in suppressing tumor growth in xenograft mouse tumor models [130]. Sorafenib, a multiple kinase inhibitor and FDA approved drug, was revealed to induce ferroptosis and exhibit anti-tumor effects in various cancer cell lines such as hepatocellular carcinoma cells, human kidney cancer cells and non-small cell lung cancer [131, 132]. Whereas, the high risk for cardiovascular complications should be aware when these ferroptosis-inducing agents are clinically utilized in cancer treatment. Duran et al. showed that sorafenib can cause cardiotoxicity through inducing myocyte necrosis, which dramatically increases mortality in the setting of MI [133]. A clinical study reported that the use of sorafenib for advanced renal cell carcinoma induced acute MI [134]. Moreover, sorafenibassociated heart failure was reported in treatment of advanced stage hepatocellular carcinoma [135]. Currently, the molecular mechanisms of FIN agents posing a potential risk to cardiovascular system still remain elusive and require further investigation. Likely, the induction of ferroptosis by FIN agents may promote the synchronized RN and inflammation reaction, resulting in cardiovascular cells dysfunction. Therefore, the treatment of cancers based on ferroptosis induction should be cautious to reduce damage to the cardiovascular system.

\section{CONCLUSIONS AND FUTURE PERSPECTIVES}

In recent years, studies on the role of ferroptosis in various diseases such as cancer and CVDs receive extensive attention. PUFA or LPO and excessive iron accumulation are obvious features of ferroptosis, that cannot be observed in other forms of cell death. Moreover, ferroptosis is tightly associated with various biological processes, including amino acid, iron, and PUFA metabolism, and the biosynthesis of glutathione, phospholipids, and coenzyme Q10 [27]. Therefore, ferroptosis as a nexus linking oxidative stress and inflammation is inevitable to play important roles in the pathogenesis of CVDs such as atherosclerosis, stoke, IRI, and heart failure. Currently, some approved and safe molecules that regulate ferroptosis by directly or indirectly targeting iron metabolism and LPO are already used to ameliorate cardiovascular injury, doxorubicin cardiotoxicity, and other diseases $[111,112]$. Cardiac pathological conditions such as acute myocardial infarct (AMI) and I/R injury are closely bound up with ROS overproduction and further influence ferroptosis [136]. Ferroptosis studies in heart diseases validate existing mechanisms, suggesting that disturbance of ferroptosis might be beneficial to 
alleviate myocardial cell death and dysfunction in diseases like iron overload cardiomyopathy and I/R injury.

Collectively, ferroptosis might be a sensor in response to the pathophysiological alterations that disrupt the iron, ROS and inflammation homeostasis of our cardiovascular system. The study of ferroptosis is still undergoing and there are many unsolved mysteries such as the drivers of LPO, the subcellular location of lethal lipid peroxides and the exact mechanism of LPO leading to cell death [137]. Answers to these questions will provide novel insights into the mechanisms of ferroptotic cell death and associated human diseases, as well as new therapeutic strategies for CVDs treatment.

\section{REFERENCES}

1. Moris D, Spartalis M, Spartalis E, Karachaliou GS, Karaolanis Gl, Tsourouflis G, et al. The role of reactive oxygen species in the pathophysiology of cardiovascular diseases and the clinical significance of myocardial redox. Ann Transl Med. 2017;5:326

2. Fassett RG, Coombes JS. Astaxanthin, oxidative stress, inflammation and cardiovascular disease. Future Cardiol. 2009;5:333-42.

3. Rizvi M, Pathak D, Freedman JE, Chakrabarti S. CD40-CD40 ligand interactions in oxidative stress, inflammation and vascular disease. Trends Mol Med. 2008; 14:530-8.

4. Gao L, Laude K, Cai H. Mitochondrial pathophysiology, reactive oxygen species, and cardiovascular diseases. Vet Clin North Am Small Anim Pract. 2008;38:137-55.

5. Luscher TF. Ageing, inflammation, and oxidative stress: final common pathways of cardiovascular disease. Eur Heart J. 2015;36:3381-3.

6. Hussain T, Tan B, Yin YL, Blachier F, Tossou MCB, Rahu N. Oxidative stress and inflammation: what polyphenols can do for us? Oxid Med Cell Longev. 2016;2016:7432797.

7. Reuter S, Gupta SC, Chaturvedi MM, Aggarwal BB. Oxidative stress, inflammation, and cancer How are they linked? Free Radic Biol Med. 2010;49:1603-16.

8. Goitre L, Balzac F, Degani S, Degan P, Marchi S, Pinton P, et al. KRIT1 regulates the homeostasis of intracellular reactive oxygen species. PLoS ONE. 2010;5: e11786.

9. Dickinson BC, Chang CJ. Chemistry and biology of reactive oxygen species in signaling or stress responses. Nat Chem Biol. 2011;7:504-11.

10. Kobayashi M, Suhara T, Baba Y, Kawasaki NK, Higa JK, Matsui T. Pathological roles of iron in cardiovascular disease. Curr Drug Targets. 2018;19:1068-76.

11. Ray PD, Huang BW, Tsuji Y. Reactive oxygen species (ROS) homeostasis and redox regulation in cellular signaling. Cell Signal. 2012;24:981-90.

12. Van Opdenbosch N, Lamkanfi M. Caspases in cell death, inflammation, and disease. Immunity. 2019;50:1352-64.

13. Yang WS, Stockwell BR. Ferroptosis: death by lipid peroxidation. Trends Cell Biol. 2016;26:165-76.

14. Friedmann Angeli JP, Krysko DV, Conrad M. Ferroptosis at the crossroads of cancer-acquired drug resistance and immune evasion. Nat Rev Cancer. 2019;19:405-14.

15. Jiang L, Kon N, Li TY, Wang SJ, Su T, Hibshoosh H, et al. Ferroptosis as a p53mediated activity during tumour suppression. Nature. 2015;520:57-62.

16. Hassannia B, Vandenabeele P, Vanden Berghe T. Targeting ferroptosis to iron out cancer. Cancer Cell. 2019;35:830-49.

17. Bai T, Li M, Liu Y, Qiao Z, Wang Z. Inhibition of ferroptosis alleviates atherosclerosis through attenuating lipid peroxidation and endothelial dysfunction in mouse aortic endothelial cell. Free Radic Biol Med. 2020;160:92-102.

18. Yamada N, Karasawa T, Wakiya T, Sadatomo A, Ito H, Kamata R, et al. Iron overload as a risk factor for hepatic ischemia-reperfusion injury in liver transplantation: potential role of ferroptosis. Am J Transplant. 2020;20:1606-18.

19. Fang $X X$, Wang $H$, Han D, Xie EJ, Yang X, Wei JY, et al. Ferroptosis as a target for protection against cardiomyopathy. Proc Natl Acad Sci USA. 2019;116:2672-80.

20. Fang $X$, Cai Z, Wang $H$, Han D, Cheng $Q$, Zhang $P$, et al. Loss of cardiac ferritin $H$ facilitates cardiomyopathy via Slc7a11-mediated ferroptosis. Circ Res. 2020;127:486-501.

21. Liu B, Zhao C, Li H, Chen X, Ding Y, Xu S. Puerarin protects against heart failure induced by pressure overload through mitigation of ferroptosis. Biochem Biophys Res Commun. 2018;497:233-40.

22. Angeli JPF, Shah R, Pratt DA, Conrad M. Ferroptosis inhibition: mechanisms and opportunities. Trends Pharmacol Sci. 2017;38:489-98.

23. Dixon SJ, Lemberg KM, Lamprecht MR, Skouta R, Zaitsev EM, Gleason CE, et al. Ferroptosis: an iron-dependent form of nonapoptotic cell death. Cell. 2012;149:1060-72.
24. Dolma S, Lessnick SL, Hahn WC, Stockwell BR. Identification of genotypeselective antitumor agents using synthetic lethal chemical screening in engineered human tumor cells. Cancer Cell. 2003;3:285-96.

25. Kayagaki N, Stowe IB, Lee BL, O'Rourke K, Anderson K, Warming S, et al. Caspase11 cleaves gasdermin $D$ for non-canonical inflammasome signalling. Nature. 2015;526:666-71.

26. Ooko E, Saeed ME, Kadioglu O, Sarvi S, Colak M, Elmasaoudi K, et al. Artemisinin derivatives induce iron-dependent cell death (ferroptosis) in tumor cells. Phytomedicine. 2015;22:1045-54.

27. Stockwell BR, Angeli JPF, Bayir H, Bush Al, Conrad M, Dixon SJ, et al. Ferroptosis: a regulated cell death nexus linking metabolism, redox biology, and disease. Cell. 2017;171:273-85.

28. Qiu Y, Cao Y, Cao W, Jia Y, Lu N. The application of ferroptosis in diseases. Pharmacol Res. 2020;159:104919.

29. Maher $P$. The effects of stress and aging on glutathione metabolism. Ageing Res Rev. 2005;4:288-314.

30. Barrera G. Oxidative stress and lipid peroxidation products in cancer progression and therapy. ISRN Oncol. 2012;2012:137289.

31. Barrera G, Pizzimenti S, Dianzani MU. Lipid peroxidation: control of cell proliferation, cell differentiation and cell death. Mol Asp Med. 2008;29:1-8.

32. Seiler A, Schneider M, Forster H, Roth S, Wirth EK, Culmsee $C$, et al. Glutathione peroxidase 4 senses and translates oxidative stress into 12/15-lipoxygenase dependent- and AIF-mediated cell death. Cell Metab. 2008;8:237-48.

33. Yang WS, Kim KJ, Gaschler MM, Patel M, Shchepinov MS, Stockwell BR. Peroxidation of polyunsaturated fatty acids by lipoxygenases drives ferroptosis. Proc Natl Acad Sci USA. 2016;113:E4966-75.

34. Yan N, Zhang JJ. The emerging roles of ferroptosis in vascular cognitive impairment. Front Neurosci. 2019;13:811.

35. Dix TA, Aikens J. Mechanisms and biological relevance of lipid peroxidation initiation. Chem Res Toxicol. 1993;6:2-18.

36. Doll S, Proneth B, Tyurina YY, Panzilius E, Kobayashi S, Ingold I, et al. ACSL4 dictates ferroptosis sensitivity by shaping cellular lipid composition. Nat Chem Biol. 2017;13:91-8.

37. Mou Y, Wang J, Wu J, He D, Zhang C, Duan C, et al. Ferroptosis, a new form of cell death: opportunities and challenges in cancer. J Hematol Oncol. 2019;12:34.

38. Shah R, Shchepinov MS, Pratt DA. Resolving the role of lipoxygenases in the initiation and execution of ferroptosis. ACS Cent Sci. 2018;4:387-96.

39. Shintoku R, Takigawa Y, Yamada K, Kubota C, Yoshimoto Y, Takeuchi T, et al. Lipoxygenase-mediated generation of lipid peroxides enhances ferroptosis induced by erastin and RSL3. Cancer Sci. 2017;108:2187-94.

40. Mao HM, Zhao YH, Li HX, Lei L. Ferroptosis as an emerging target in inflammatory diseases. Prog Biophys Mol Biol. 2020;155:20-8.

41. Bersuker K, Hendricks JM, Li Z, Magtanong L, Ford B, Tang PH, et al. The CoQ oxidoreductase FSP1 acts parallel to GPX4 to inhibit ferroptosis. Nature. 2019;575:688-92.

42. Doll S, Freitas FP, Shah R, Aldrovandi M, da Silva MC, Ingold I, et al. FSP1 is a glutathione-independent ferroptosis suppressor. Nature. 2019;575:693-8.

43. Hadian K. Ferroptosis suppressor protein 1 (FSP1) and coenzyme Q10 cooperatively suppress ferroptosis. Biochemistry. 2020;59:637-8.

44. Garcia-Bermudez J, Baudrier L, Bayraktar EC, Shen Y, La K, Guarecuco R, et al. Squalene accumulation in cholesterol auxotrophic lymphomas prevents oxidative cell death. Nature. 2019;567:118-22.

45. Dai E, Zhang W, Cong D, Kang R, Wang J, Tang D. AIFM2 blocks ferroptosis independent of ubiquinol metabolism. Biochem Biophys Res Commun. 2020;523:966-71.

46. Sarhan M, Land WG, Tonnus W, Hugo CP, Linkermann A. Origin and consequences of necroinflammation. Physiol Rev. 2018;98:727-80.

47. Linkermann A. Death and fire-the concept of necroinflammation. Cell Death Differ. 2019;26:1-3.

48. Linkermann A, Stockwell BR, Krautwald S, Anders HJ. Regulated cell death and inflammation: an auto-amplification loop causes organ failure. Nat Rev Immunol. 2014;14:759-67.

49. Land WG, Agostinis $P$, Gasser S, Garg AD, Linkermann A. Transplantation and damage-associated molecular patterns (DAMPs). Am J Transpl. 2016;16:3338-61.

50. Proneth B, Conrad M. Ferroptosis and necroinflammation, a yet poorly explored link. Cell Death Differ. 2019;26:14-24.

51. Gong T, Liu L, Jiang W, Zhou R. DAMP-sensing receptors in sterile inflammation and inflammatory diseases. Nat Rev Immunol. 2020;20:95-112.

52. Pandolfi F, Altamura S, Frosali S, Conti P. Key role of DAMP in inflammation, cancer, and tissue repair. Clin Ther 2016;38:1017-28.

53. Martinez MA, Rodriguez JL, Lopez-Torres B, Martinez M, Martinez-Larranaga MR, Anadon A. Oxidative stress and related gene expression effects of cyfluthrin in human neuroblastoma SH-SY5Y cells: protective effect of melatonin. Environ Res. 2019;177:108579 
54. Mak TW, Grusdat M, Duncan GS, Dostert C, Nonnenmacher Y, Cox M, et al. Glutathione primes $T$ cell metabolism for inflammation. Immunity. 2017;46:675-89.

55. Chen CJ, Huang HS, Chang WC. Depletion of phospholipid hydroperoxide glutathione peroxidase up-regulates arachidonate metabolism by $12 \mathrm{~S}$ lipoxygenase and cyclooxygenase 1 in human epidermoid carcinoma A431 cells. FASEB J. 2003;17:1694-6.

56. Sakamoto $\mathrm{H}$, Imai H, Nakagawa Y. Involvement of phospholipid hydroperoxide glutathione peroxidase in the modulation of prostaglandin D2 synthesis. J Biol Chem. 2000;275:40028-35.

57. Li C, Deng XB, Xie XW, Liu Y, Angeli JPF, Lai LH. Activation of glutathione peroxidase 4 as a novel anti-inflammatory strategy. Front Pharmacol. 2018;9:1120

58. Suzuki H, Kayama Y, Sakamoto M, luchi H, Shimizu I, Yoshino T, et al. Arachidonate 12/15-lipoxygenase-induced inflammation and oxidative stress are involved in the development of diabetic cardiomyopathy. Diabetes. 2015:64:618-30

59. Calder PC. Polyunsaturated fatty acids, inflammation, and immunity. Lipids. 2001;36:1007-24.

60. Sanchez A, Contreras C, Villalba N, Martinez P, Martinez AC, Briones A, et al. Altered arachidonic acid metabolism via COX-1 and COX-2 contributes to the endothelial dysfunction of penile arteries from obese Zucker rats. Br J Pharmacol. 2010;159:604-16.

61. Mashima R, Okuyama T. The role of lipoxygenases in pathophysiology; new insights and future perspectives. Redox Biol. 2015;6:297-310.

62. Pearson T, Warren AY, Barrett DA, Khan RN. Detection of EETs and HETEgenerating cytochrome P-450 enzymes and the effects of their metabolites on myometrial and vascular function. Am J Physiol Endocrinol Metab. 2009;297: E647-56.

63. Zhou Y, Que KT, Zhang Z, Yi ZJ, Zhao PX, You Y, et al. Iron overloaded polarizes macrophage to proinflammation phenotype through ROS/acetyl-p53 pathway. Cancer Med. 2018;7:4012-22.

64. Handa P, Thomas S, Morgan-Stevenson V, Maliken BD, Gochanour E, Boukhar S, et al. Iron alters macrophage polarization status and leads to steatohepatitis and fibrogenesis. J Leukoc Biol. 2019;105:1015-26.

65. Islam S, Jarosch S, Zhou J, Parquet MD, Toguri JT, Colp P, et al. Anti-inflammatory and anti-bacterial effects of iron chelation in experimental sepsis. J Surg Res. 2016;200:266-73.

66. Thorburn T, Aali M, Kostek L, LeTourneau-Paci C, Colp P, Zhou J, et al. Antiinflammatory effects of a novel iron chelator, DIBI, in experimental sepsis. Clin Hemorheol Microcirc. 2017;67:241-50.

67. Liu J, Thewke DP, Su YR, Linton MF, Fazio S, Sinensky MS. Reduced macrophage apoptosis is associated with accelerated atherosclerosis in lowdensity lipoprotein receptor-null mice. Arterioscler Thromb Vasc Biol. 2005;25:174-9.

68. Xiong YY, Yu Y, Montani JP, Yang ZH, Ming XF. Arginase-II induces vascular smooth muscle cell senescence and apoptosis through p66Shc and p53 independently of its L-arginine ureahydrolase activity: implications for atherosclerotic plaque vulnerability. J Am Heart Assoc.2013;2:e00096

69. Rayner KJ. Cell death in the vessel wall the good, the bad, the ugly. Arterioscler Thromb Vasc Biol. 2017:37:E75-81.

70. Yin $Y, L i X Y$, Sha $X J, X i ~ H, L i ~ Y F$, Shao $Y$, et al. Early hyperlipidemia promotes endothelial activation via a caspase-1-sirtuin 1 pathway. Arterioscler Thromb Vasc Biol. 2015;35:804-16.

71. Gage J, Hasu M, Thabet M, Whitman SC. Caspase-1 deficiency decreases atherosclerosis in apolipoprotein E-null mice. Can J Cardiol. 2012;28:222-9.

72. Karunakaran D, Geoffrion M, Wei L, Gan W, Richards L, Shangari P, et al. Targeting macrophage necroptosis for therapeutic and diagnostic interventions in atherosclerosis. Sci Adv. 2016;2:e1600224.

73. Guo Z, Ran Q, Roberts LJ 2nd, Zhou L, Richardson A, Sharan C, et al. Suppression of atherogenesis by overexpression of glutathione peroxidase-4 in apolipoprotein E-deficient mice. Free Radic Biol Med. 2008:44:343-52.

74. Dabbagh AJ, Mannion T, Lynch SM, Frei B. The effect of iron overload on rat plasma and liver oxidant status in vivo. Biochem J. 1994;300:799-803.

75. Swain J, Gutteridge JM. Prooxidant iron and copper, with ferroxidase and xanthine oxidase activities in human atherosclerotic material. FEBS Lett. 1995;368:513-5.

76. Shah SV, Alam MG. Role of iron in atherosclerosis. Am J Kidney Dis. 2003;41: S80-3.

77. Feng H, Schorpp K, Jin J, Yozwiak CE, Hoffstrom BG, Decker AM, et al. Transferrin receptor is a specific ferroptosis marker. Cell Rep. 2020:30:3411-23.e3417.

78. de Valk B, Marx JJ. Iron, atherosclerosis, and ischemic heart disease. Arch Intern Med. 1999;159:1542-8.

79. Martinet W, Coornaert I, Puylaert P, De Meyer GRY. Macrophage death as a pharmacological target in atherosclerosis. Front Pharmacol. 2019;10:306
80. Yellon DM, Hausenloy DJ. Myocardial reperfusion injury. $\mathrm{N}$ Engl J Med. 2007;357:1121-35.

81. Bolli R. Oxygen-derived free radicals and myocardial reperfusion injury: an overview. Cardiovasc Drugs Ther. 1991;5:249-68.

82. Garcia-Gil FA, Gonzalvo E, Garcia-Garcia JJ, Albendea CD, Guemes A, TomeZelaya $\mathrm{E}$, et al. Lipid peroxidation in ischemia-reperfusion oxidative injury of the graft preserved in Celsior and University of Wisconsin solutions on a pig pancreas transplantation model. Transplant Proc. 2006;38:2595-9.

83. Lucas DT, Szweda LI. Cardiac reperfusion injury: aging, lipid peroxidation, and mitochondrial dysfunction. Proc Natl Acad Sci USA. 1998;95:510-4.

84. Linkermann A, Brasen JH, Darding M, Jin MK, Sanz AB, Heller JO, et al. Two independent pathways of regulated necrosis mediate ischemia-reperfusion injury. Proc Natl Acad Sci USA. 2013;110:12024-9.

85. Angeli JPF, Schneider M, Proneth B, Tyurina YY, Tyurin VA, Hammond VJ, et al. Inactivation of the ferroptosis regulator $\mathrm{Gpx} 4$ triggers acute renal failure in mice. Nat Cell Biol. 2014;16:1180-20.

86. Hofmans S, Vanden Berghe T, Devisscher L, Hassannia B, Lyssens S, Joossens J, et al. Novel ferroptosis inhibitors with improved potency and ADME properties. J Med Chem. 2016;59:2041-53.

87. Li Q, Han X, Lan X, Gao Y, Wan J, Durham F, et al. Inhibition of neuronal ferroptosis protects hemorrhagic brain. JCI Insight. 2017;2:e90777.

88. Gao MH, Monian P, Quadri N, Ramasamy R, Jiang XJ. Glutaminolysis and transferrin regulate ferroptosis. Mol Cell. 2015;59:298-308.

89. Li WJ, Feng GS, Gauthier JM, Lokshina I, Higashikubo R, Evans S, et al. Ferroptotic cell death and TLR4/Trif signaling initiate neutrophil recruitment after heart transplantation. J Clin Invest. 2019;129:2293-304.

90. Tonnus W, Linkermann A. "Death is my Heir"-ferroptosis connects cancer pharmacogenomics and ischemia-reperfusion injury. Cell Chem Biol. 2016;23:202-3.

91. Linkermann A, Skouta R, Himmerkus N, Mulay SR, Dewitz C, De Zen F, et al. Synchronized renal tubular cell death involves ferroptosis. Proc Natl Acad Sci USA. 2014;111:16836-41.

92. Kontoghiorghes GJ, Kolnagou A, Skiada A, Petrikkos G. The role of iron and chelators on infections in iron overload and non iron loaded conditions: prospects for the design of new antimicrobial therapies. Hemoglobin. 2010;34:227-39.

93. Cottin Y, Doise JM, Maupoil V, Tanniere-Zeller M, Dalloz F, Maynadie M, et al. Plasma iron status and lipid peroxidation following thrombolytic therapy for acute myocardial infarction. Fundam Clin Pharmacol. 1998;12:236-41.

94. Zhao G, Ayene IS, Fisher AB. Role of iron in ischemia-reperfusion oxidative injury of rat lungs. Am J Respir Cell Mol Biol. 1997;16:293-9.

95. Alim I, Caulfield JT, Chen YX, Swarup V, Geschwind DH, Ivanova E, et al. Selenium drives a transcriptional adaptive program to block ferroptosis and treat stroke. Cell. 2019;177:1262-79.e1225.

96. Munoz M, Villar I, Garcia-Erce JA. An update on iron physiology. World J Gastroenterol. 2009;15:4617-26.

97. Poss KDTS. Heme oxygenase 1 is required for mammalian iron reutilization. Proc Natl Acad Sci USA. 1997;20:10919-24.

98. Lipscomb DC, Gorman LG, Traystman RJ, Hurn PD. Low molecular weight iron in cerebral ischemic acidosis in vivo. Stroke. 1998;29:487-92. discussion 493.

99. Selim MH, Ratan RR. The role of iron neurotoxicity in ischemic stroke. Ageing Res Rev. 2004;3:345-53.

100. Ishimaru $\mathrm{H}$, Ishikawa $\mathrm{K}$, Ohe $\mathrm{Y}$, Takahashi $A$, Tatemoto $\mathrm{K}$, Maruyama $\mathrm{Y}$. Activation of iron handling system within the gerbil hippocampus after cerebral ischemia. Brain Res. 1996;726:23-30.

101. Chang CY, Lai YC, Cheng TJ, Lau MT, Hu ML. Plasma levels of antioxidant vitamins, selenium, total sulfhydryl groups and oxidative products in ischemicstroke patients as compared to matched controls in Taiwan. Free Radic Res. 1998;28:15-24.

102. Lee WC, Wong HY, Chai YY, Shi CW, Amino N, Kikuchi S, et al. Lipid peroxidation dysregulation in ischemic stroke: plasma $4-\mathrm{HNE}$ as a potential biomarker? Biochem Biophys Res Commun. 2012;425:842-7.

103. Sharpe PC, Mulholland C, Trinick T. Ascorbate and malondialdehyde in stroke patients. Ir J Med Sci. 1994;163:488-91.

104. Ferretti G, Bacchetti T, Masciangelo S, Nanetti L, Mazzanti L, Silvestrini M, et al. Lipid peroxidation in stroke patients. Clin Chem Lab Med. 2008;46:113-7.

105. Baba Y, Higa JK, Shimada BK, Horiuchi KM, Suhara T, Kobayashi M, et al. Protective effects of the mechanistic target of rapamycin against excess iron and ferroptosis in cardiomyocytes. Am J Physiol Heart Circ Physiol. 2018;314: H659-68.

106. Conrad M, Proneth B. Broken hearts: Iron overload, ferroptosis and cardiomyopathy. Cell Res. 2019;29:263-4.

107. Bai YT, Chang R, Wang H, Xiao FJ, Ge RL, Wang LS. ENPP2 protects cardiomyocytes from erastin-induced ferroptosis. Biochem Biophys Res Commun. 2018;499:44-51. 
108. Chen XQ, Xu SD, Zhao CX, Liu B. Role of TLR4/NADPH oxidase 4 pathway in promoting cell death through autophagy and ferroptosis during heart failure. Biochem Biophys Res Commun. 2019;516:37-43.

109. Kang R, Tang D. Autophagy and ferroptosis - what's the connection? Curr Pathobiol Rep. 2017;5:153-9.

110. Gammella E, Recalcati S, Cairo G. Dual role of ROS as signal and stress agents: iron tips the balance in favor of toxic effects. Oxid Med Cell Longev. 2016;2016:8629024.

111. Gujja P, Rosing DR, Tripodi DJ, Shizukuda Y. Iron overload cardiomyopathy: better understanding of an increasing disorder. J Am Coll Cardiol. 2010;56:1001-12.

112. Dendorfer A, Heidbreder M, Hellwig-Burgel T, Johren O, Qadri F, Dominiak P. Deferoxamine induces prolonged cardiac preconditioning via accumulation of oxygen radicals. Free Radic Biol Med. 2005;38:117-24.

113. Zhang WJ, Wei H, Frei B. The iron chelator, desferrioxamine, reduces inflammation and atherosclerotic lesion development in experimental mice. Exp Biol Med. 2010;235:633-41.

114. Duffy SJ, Biegelsen ES, Holbrook M, Russell JD, Gokce N, Keaney JF Jr., et al. Iron chelation improves endothelial function in patients with coronary artery disease. Circulation. 2001;103:2799-804.

115. Abdul Y, Li W, Ward R, Abdelsaid M, Hafez S, Dong G, et al. Deferoxamine treatment prevents post-stroke vasoregression and neurovascular unit remodeling leading to improved functional outcomes in type 2 male diabetic rats: role of endothelial ferroptosis. Transl Stroke Res. 2020;12:615-30.

116. Wortmann M, Schneider M, Pircher J, Hellfritsch J, Aichler M, Vegi N, et al. Combined deficiency in glutathione peroxidase 4 and vitamin $\mathrm{E}$ causes multiorgan thrombus formation and early death in mice. Circ Res. 2013;113:408-17.

117. Hu CL, Nydes M, Shanley KL, Morales Pantoja IE, Howard TA, Bizzozero OA. Reduced expression of the ferroptosis inhibitor glutathione peroxidase-4 in multiple sclerosis and experimental autoimmune encephalomyelitis. J Neurochem. 2019;148:426-39.

118. Kang R, Zeng L, Zhu S, Xie Y, Liu J, Wen Q, et al. Lipid peroxidation drives gasdermin D-mediated pyroptosis in lethal polymicrobial sepsis. Cell Host Microbe. 2018;24:e104.

119. Yoo SE, Chen L, Na R, Liu Y, Rios C, Van Remmen H, et al. Gpx4 ablation in adult mice results in a lethal phenotype accompanied by neuronal loss in brain. Free Radic Biol Med. 2012;52:1820-7.

120. Sengupta A, Lichti UF, Carlson BA, Cataisson C, Ryscavage AO, Mikulec C, et al. Targeted disruption of glutathione peroxidase 4 in mouse skin epithelial cells impairs postnatal hair follicle morphogenesis that is partially rescued through inhibition of COX-2. J Invest Dermatol. 2013;133:1731-41.

121. Prasad A, Andrews NP, Padder FA, Husain M, Quyyumi AA. Glutathione reverses endothelial dysfunction and improves nitric oxide bioavailability. J Am Coll Cardiol. 1999;34:507-14.

122. Dabkowski ER, Williamson $\mathrm{CL}$, Hollander JM. Mitochondria-specific transgenic overexpression of phospholipid hydroperoxide glutathione peroxidase (GPx4) attenuates ischemia/reperfusion-associated cardiac dysfunction. Free Radic Biol Med. 2008;45:855-65.

123. Devaraj S, Jialal I. Failure of vitamin $\mathrm{E}$ in clinical trials: is gamma-tocopherol the answer? Nutr Rev. 2005;63:290-3.

124. Hinman A, Holst CR, Latham JC, Bruegger JJ, Ulas G, McCusker KP, et al. Vitamin $E$ hydroquinone is an endogenous regulator of ferroptosis via redox control of 15-lipoxygenase. PLoS ONE. 2018;13:e0201369.

125. Upston JM, Kritharides L, Stocker R. The role of vitamin E in atherosclerosis. Prog Lipid Res. 2003;42:405-22.

126. Zilka O, Shah R, Li B, Friedmann Angeli JP, Griesser M, Conrad M, et al. On the mechanism of cytoprotection by ferrostatin-1 and liproxstatin-1 and the role of lipid peroxidation in ferroptotic cell death. ACS Cent Sci. 2017;3:232-43.

127. Feng Y, Madungwe NB, Imam Aliagan AD, Tombo N, Bopassa JC. Liproxstatin-1 protects the mouse myocardium against ischemia/reperfusion injury by decreasing VDAC1 levels and restoring GPX4 levels. Biochem Biophys Res Commun. 2019;520:606-11.

128. Chen G, Guo G, Zhou X, Chen H. Potential mechanism of ferroptosis in pancreatic cancer. Oncol Lett. 2020;19:579-87.

129. Xie $Y$, Hou $W$, Song $X, Y u$, Huang J, Sun $X$, et al. Ferroptosis: process and function. Cell Death Differ. 2016;23:369-79.

130. Yang WS, SriRamaratnam R, Welsch ME, Shimada K, Skouta R, Viswanathan VS, et al. Regulation of ferroptotic cancer cell death by GPX4. Cell. 2014;156:317-31.

131. Lachaier E, Louandre C, Godin C, Saidak Z, Baert M, Diouf M, et al. Sorafenib induces ferroptosis in human cancer cell lines originating from different solid tumors. Anticancer Res. 2014;34:6417-22.
132. Li Y, Yan HY, Xu XM, Liu HB, Wu C, Zhao L. Erastin/sorafenib induces cisplatinresistant non-small cell lung cancer cell ferroptosis through inhibition of the Nrf2/xCT pathway. Oncol Lett. 2020;19:323-33.

133. Duran JM, Makarewich CA, Trappanese D, Gross $P$, Husain S, Dunn J, et al. Sorafenib cardiotoxicity increases mortality after myocardial infarction. Circ Res. 2014;114:1700-12.

134. Arima Y, Oshima S, Noda K, Fukushima H, Taniguchi I, Nakamura S, et al. Sorafenib-induced acute myocardial infarction due to coronary artery spasm. J Cardiol. 2009;54:512-5.

135. Wu C, Shemisa K. Sorafenib-associated heart failure complicated by cardiogenic shock after treatment of advanced stage hepatocellular carcinoma: a clinical case discussion. Case Rep Cardiol. 2017;2017:7065759.

136. Cadenas S. ROS and redox signaling in myocardial ischemia-reperfusion injury and cardioprotection. Free Radic Biol Med. 2018;117:76-89.

137. Feng $H$, Stockwell BR. Unsolved mysteries: how does lipid peroxidation cause ferroptosis? PLoS Biol. 2018;16:e2006203.

\section{AUTHOR CONTRIBUTIONS}

$Y Y, Y Y, N F L, W Y Q, Z X L Q L, L P$, and $X Y Y$ performed substantial contributions to conception, design, and assembled the figure. $Y Y, C X Y, S G D, L Y R, Q L, L P$, and $X Y Y$ drafted the article or revised it critically for important intellectual content and approved the final version to be published.

\section{FUNDING STATEMENT}

This work was supported by the Natural Science Foundation of Shaanxi Province (Grant no. 2020JQ-611), Special Scientific Research Plan for Emergency Public Health Safety 2020 of Shaanxi Provincial Department of Education (Grant no. 20JG034), Scientific Research Foundation 2019 for the Returned Overseas Scholars of Shaanxi Province and High-level Talent Programs of Shaanxi Province.

\section{ETHICAL STATEMENT}

Not applicable.

\section{COMPETING INTERESTS}

The authors declare no competing interests.

\section{ADDITIONAL INFORMATION}

Correspondence and requests for materials should be addressed to L.Q., P.L. or Y.X.

Reprints and permission information is available at http://www.nature.com/ reprints

Publisher's note Springer Nature remains neutral with regard to jurisdictional claims in published maps and institutional affiliations.

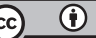

Open Access This article is licensed under a Creative Commons Attribution 4.0 International License, which permits use, sharing, adaptation, distribution and reproduction in any medium or format, as long as you give appropriate credit to the original author(s) and the source, provide a link to the Creative Commons license, and indicate if changes were made. The images or other third party material in this article are included in the article's Creative Commons license, unless indicated otherwise in a credit line to the material. If material is not included in the article's Creative Commons license and your intended use is not permitted by statutory regulation or exceeds the permitted use, you will need to obtain permission directly from the copyright holder. To view a copy of this license, visit http://creativecommons. org/licenses/by/4.0/.

(c) The Author(s) 2021 